\title{
Modular Propagation of Epileptiform Activity: Evidence for an Inhibitory Veto in Neocortex
}

\author{
Andrew J. Trevelyan, ${ }^{1,2}$ David Sussillo, ${ }^{1}$ Brendon 0. Watson, ${ }^{1}$ and Rafael Yuste ${ }^{1}$ \\ ${ }^{1}$ Howard Hughes Medical Institute, Department of Biological Sciences, Columbia University, New York, New York 10027, and ${ }^{2}$ School of Neurology, \\ Neurobiology, and Psychiatry, The Medical School, University of Newcastle, Newcastle NE2 4HH, United Kingdom
}

What regulates the spread of activity through cortical circuits? We present here data indicating a pivotal role for a vetoing inhibition restraining modules of pyramidal neurons. We combined fast calcium imaging of network activity with whole-cell recordings to examine epileptiform propagation in mouse neocortical slices. Epileptiform activity was induced by washing $\mathrm{Mg}^{2+}$ ions out of the slice. Pyramidal cells receive barrages of inhibitory inputs in advance of the epileptiform wave. The inhibitory barrages are effectively nullified at low doses of picrotoxin $(2.5-5 \mu \mathrm{M})$. When present, however, these inhibitory barrages occlude an intense excitatory synaptic drive that would normally exceed action potential threshold by approximately a factor of 10 . Despite this level of excitation, the inhibitory barrages suppress firing, thereby limiting further neuronal recruitment to the ictal event. Pyramidal neurons are recruited to the epileptiform event once the inhibitory restraint fails and are recruited in spatially clustered populations (150-250 $\mu \mathrm{m}$ diameter). The recruitment of the cells within a given module is virtually simultaneous, and thus epileptiform events progress in intermittent $(0.5-1 \mathrm{~Hz})$ steps across the cortical network. We propose that the interneurons that supply the vetoing inhibition define these modular circuit territories.

Key words: epilepsy; seizure; calcium imaging; inhibition; basket cell; pyramidal cell

\section{Introduction}

Normal cortical function depends critically on proper regulation of the lateral spread of activity. Cortical processing occurs within the context of topographic maps (Hubel and Wiesel, 1977), the precision of which requires that activity is kept focused. Furthermore, if activity is allowed to spread unrestrained, epileptic seizures ensue. Clearly, the lateral spread of activity is an important issue for both normal physiology and pathophysiology in neocortex.

Focal neocortical epilepsy is characterized by repeated, localized "interictal" events and rarer, full "ictal" events in which the activity breaks away from the restricted focus (Gastaut and Broughton, 1972). Interictal activity can be recorded electrophysiologically, yet crucially, it is clinically silent: it provokes no outward physical manifestations, and the patient is unaware of the activity (Gastaut and Broughton, 1972). Only when activity breaks away from the ictogenic focus is the epilepsy manifest. Thus, if the activity can be restricted to the pathological part of the network, then the epilepsy may be controlled.

Yet how activity is contained remains a mystery. Intermediate-

Received June 30, 2006; revised Sept. 12, 2006; accepted 0ct. 19, 2006.

This work was supported by the Epilepsy Foundation of America, the National Institute of Neurological Disorders and Stroke, the National Eye Institute, the Human Frontiers Science Project, and a Biophysics Training grant (GM008281). We thank Morgane Jego, Jason Maclean, and Miles Whittington for their helpful and supportive comments on previous drafts of this manuscript. A.J.T. particularly thanks Miles Whittington and Fiona Le Beau for the loan of various equipment and consumables.

Correspondence should be addressed to Andrew J. Trevelyan, School of Neurology, Neurobiology, and Psychiatry, The Medical School, University of Newcastle, Framlington Place, Newcastle NE2 4HH, UK. E-mail: andytrev@gmail.com.

DOI:10.1523/JNEUROSCI.2787-06.2006

Copyright $\odot 2006$ Society for Neuroscience $\quad$ 0270-6474/06/2612447-09\$15.00/0 range, excitatory, lateral connections (400-1000 $\mu \mathrm{m})$ show topographic specificity (Gilbert and Wiesel, 1989), with regions representing similar features projecting to each other. This specificity, however, appears lacking at shorter distances (Malach, 1992; Malach et al., 1993; Douglas and Martin, 2004), implying that topographic precision does not arise simply from the pattern of excitatory connections. Rather, there is likely to be a contribution from inhibitory interneurons as further suggested by evidence of inhibitory restraints in neocortical focal epilepsies (Prince and Wilder, 1967; Goldensohn and Salazar, 1986; Prince and Jacobs, 1998; Schwartz and Bonhoeffer, 2001).

The precise nature of any interneuronal restraints in neocortex requires some clarification. The restraint is quickly compromised by low levels of $\mathrm{GABA}_{\mathrm{A}}$ antagonists. Progressively increasing the level of disinhibition has identified a threshold level of $\mathrm{GABA}_{\mathrm{A}}$ antagonists [ $2 \mu \mathrm{M}$ picrotoxin (PTX) (Pinto et al., 2005) or $0.4 \mu \mathrm{M}$ bicuculline methiodide (BMI) (Chagnac-Amitai and Connors, 1989)] above which epileptiform discharges spread through the tissue (Pinto et al., 2005). Surprisingly, additional increases in $\mathrm{GABA}_{\mathrm{A}}$ antagonist concentration above this threshold does not alter the measured propagation velocity (Pinto et al., 2005): studies using 2-10 $\mu \mathrm{M}$ picrotoxin (Pinto et al., 2005), 50 $\mu \mathrm{M}$ PTX (Wadman and Gutnick, 1993), and $10 \mu \mathrm{M}$ BMI (Chervin et al., 1988) all consistently reported propagation velocities of $50-90 \mathrm{~mm} / \mathrm{s}$.

Importantly, however, the zero magnesium $\left(0 \mathrm{Mg}^{2+}\right)$ model (Walther et al., 1986; Mody et al., 1987; Silva et al., 1991), in which inhibition is broadly preserved, displays robust epileptiform propagation at velocities more than two orders of magnitude slower $(\sim 200-300 \mu \mathrm{m} / \mathrm{s})$ than any of the disinhibition models (Wong and Prince, 1990). In this regard, the $0 \mathrm{Mg}^{2+}$ 
model appears to reflect better the spread of propagation in the classic description of neocortical epileptic propagation in vivo, the Jacksonian march (Jasper, 1969; Ohara et al., 2004). The slow propagation in this model, together with the fact that BMI concentrations below the threshold for epileptiform propagation can still alter the horizontal spread of activity in response to extracellular stimulation (Chagnac-Amitai and Connors, 1989), suggest that there may be important inhibitory influences that are not seen in the partial disinhibition models.

Here we examine the role of inhibition in regulating the spread of activity, using a combination of whole-cell patch-clamp recordings with fast confocal imaging of slices bulk loaded with the $\mathrm{Ca}^{2+}$ dye Oregon Green 488 BAPTA 1 (OGB1). We demonstrate that propagation in the $0 \mathrm{Mg}^{2+}$ model is slow because it is a stop-start process, with the individual steps being shaped by powerful inhibitory networks.

\section{Materials and Methods}

Slice preparation. All animal handling and experimentation were done according to National Institutes of Health guidelines. All recordings were made from slices of occipital cortex prepared acutely from postnatal day 13-49 C57BL/6 mice. The animals were anesthetized with ketaminexylazine $(10 \mathrm{mg} / \mathrm{kg})$, and the brain was removed and submerged in icecold isotonic sucrose solution (in mm: 222 sucrose, $26 \mathrm{NaHCO}_{3}, 2$ $\mathrm{MgCl}_{2}, 2.6 \mathrm{KCl}, 1.5 \mathrm{NaH}_{2} \mathrm{PO}_{4}$, and 0.05 ascorbic acid). Coronal slices, $350 \mu \mathrm{m}$ thick, were cut using a Leica (Nussloch, Germany) vibratome, and the slices were then incubated in artificial CSF (ACSF) containing 1 mM $\mathrm{Mg}^{2+}$ ions (in mu: $125 \mathrm{NaCl}, 26 \mathrm{NaHCO}_{3}, 10$ dextrose, $3.5 \mathrm{KCl}, 2$ $\mathrm{CaCl}_{2}, 1.26 \mathrm{NaH}_{2} \mathrm{PO}_{4}$, and $\left.1 \mathrm{MgSO}_{4}\right)$.

Dye loading and imaging. Slices were bulk loaded with OGB1-AM ester as follows. OGB1-AM ( $50 \mu \mathrm{g}$ vial; Invitrogen, Carlsbad, CA) was mixed with $8 \mu \mathrm{l}$ of DMSO and $2 \mu \mathrm{l}$ of pluronic acid F-127 solution (10\% in DMSO; Invitrogen). Meanwhile, the slices were preincubated at $37^{\circ} \mathrm{C}$ for $5 \mathrm{~min}$ in $3 \mathrm{ml}$ of ACSF with $8 \mu \mathrm{l}$ of Cremophor EL solution $(0.5 \%$ in DMSO; Sigma, St. Louis, MO). The OGB1/pluronic acid/DMSO mixture was then added, and the slices were incubated for an additional 30-40 min. The final concentrations were as follows: $\sim 12 \mu \mathrm{M}$ OGB1-AM ester, $0.6 \%$ DMSO, $0.002 \%$ Cremophor EL, and $0.01 \%$ pluronic F- 127 . The slices were then placed back in normal ACSF for at least $30 \mathrm{~min}$ before transferring to the recording chamber.

In most experiments, once the tissue was in the recording chamber, it was bathed briefly in sulforhodamine [SR101 (Invitrogen); $3 \mathrm{ml}$ of $1 \mu \mathrm{M}$ solution (in ACSF) and subsequently washed through for at least $15 \mathrm{~min}$ ] to label the astrocyte population (Nimmerjahn et al., 2004) (supplemental Fig. 1, available at www.jneurosci.org as supplemental material). The SR101 was visualized using epifluorescence illumination, viewed through a rhodamine filter set.

OGB1 imaging was performed using a spinning disk confocal (Solamere, Salt Lake City, UT) mounted on an Olympus Optical (Tokyo, Japan) BX/50WI upright microscope. The tissue was illuminated with a $488 \mathrm{~nm}$ laser (Coherent, Santa Clara, CA), controlled through an acoustic-optical tunable filter. Images were collected at 7.5-60 Hz with a Stanford Photonics (Palo Alto, CA) Mega camera and streamed to a raid drive (Atto Bioscience, Rockville, MD) using QED software (QED Software Solutions, Delta, British Columbia, Canada). Off-line analysis of the images was performed using NIH ImageJ and in-house software. Briefly, line scan analyses were done on movies collected using $4 \times$ or $10 \times$ objectives. A rectangular box (up to $1 \mathrm{~mm}$ long and $\sim 40-50 \mu \mathrm{m}$ width) was laid over approximately layer 5 and divided into $4 \mu \mathrm{m}$ bins aligned to the cortical columns. The average $\mathrm{Ca}^{+}$fluorescence signal for each bin was derived and plotted against time to give a line scan plot with time as the abscissa and cortical horizontal distance as the ordinate. Cellular $\mathrm{Ca}^{2+}$ signals were derived from movies taken using $20 \times$ and $40 \times$ objectives. Somas were identified as described previously (Cossart et al., 2003; Ikegaya et al., 2004; MacLean et al., 2005). The neuropil signal was subtracted from the somatic signal to derive the "center-surround" (CS) signal, which we found to be a better indicator of the activity pattern of individual cells during intense network activity. Without this simple correction, we found frequent examples when a sudden increase in the $\mathrm{Ca}^{2+}$ dye signal corresponded to a synaptic barrage but not to the firing of the cell (for additional explanation, see Fig. 7 and accompanying legend). The CS signals for individual cells were smoothed (5 point box smoothing) before taking the derivative ( $d \mathrm{CS} / d t$ plots). The range of the $d \mathrm{CS} / d t$ plots for all neurons was normalized, and spatial clustering was based on the time of maximal rate of increase of the signal.

Electrophysiology. The electrophysiological data were collected using a Heka Elektronik (Lambrecht/Pfalz, Germany) EPC10 amplifier with Patchmaster software. The bath was mounted on a Scientifica (Uckfield, East Sussex, UK) movable top plate fitted with a heater plate (Warner Instruments, Hamden, CT), and the incoming solution (perfusion at 1-3 $\mathrm{ml} / \mathrm{min}$ ) was heated by a sleeve heater element (Warner Instruments). All imaging and electrophysiological recordings were done at $33-37^{\circ} \mathrm{C}$. Whole-cell patch-clamp recordings were made using 3-7 $\mathrm{M} \Omega$ pipettes [borosilicate glass (Harvard Apparatus, Holliston, MA). We used MX130 hydraulic micromanipulators (San Diego Instruments, San Diego, CA)]. The pipette solution contained the following (in mM): $125 \mathrm{~K}$ methyl-SO 4 , 10 K-HEPES, 2.5 Mg-ATP, 6 NaCl, 5 QX-314 [N-(2,6dimethylphenylcarbamoylmethyl)triethylammonium bromide; Tocris Bioscience, Ellisville, MO], $0.3 \mathrm{Na}-\mathrm{GTP}$, and $0.5 \%$ biocytin (w/v). For current-clamp ( $\left.I_{\text {clamp }}\right)$ recordings of action potential trains, the QX-314 was omitted. For cell-attached recordings, the electrode was filled with ACSF using the same electrodes as for the whole-cell patch-clamp experiments. The electrophysiological data were analyzed off-line using Igor (WaveMetrics, Lake Oswego, OR). There were no qualitative differences between the recordings from OGB1-loaded versus unloaded slices.

Cells were routinely filled with biocytin during the recordings, and all slices were fixed and stained for biocytin. From the location of the stained cells, one could accurately determine which cortical laminas were imaged during the experiments.

\section{Results}

We targeted large layer 5 pyramidal cells for patch-clamp recordings because previous studies had identified this class of neurons as playing a key role in epileptiform spread (Connors, 1984; Telfeian and Connors, 1998). Slices were bulk loaded with the $\mathrm{Ca}^{2+}$ dye OGB1 to allow fast (17-100 ms/frame) confocal imaging in parallel with the electrophysiological recordings.

Neurons were patched while the slice was still bathed in normal ACSF, and only then was the $\mathrm{Mg}^{2+}$ washed out. This enabled us to record the earliest epileptiform activity experienced by that cortical network. We imaged initially at low magnification $(10 \times$ objective) to derive line scans through the infragranular layers (Fig. 1). These line scans consistently showed that the first full ictal events progressed in a stepwise manner (Fig. 1 Bi). Furthermore, there was a straightforward relationship between the $\mathrm{Ca}^{2+}$ signal and the electrophysiological recordings. The steps were synchronous with intense synaptic barrages on to the recorded pyramidal cell (Fig. 1 Bii).

Voltage-clamp $\left(V_{\text {clamp }}\right)$ recordings showed that the sign (inhibition or excitation) of the barrage had a clear topographic relationship to the location of network activity: upstream activity provoked predominantly inhibitory barrages (Fig. 1 Bii), whereas once the wave reached the recorded cell, only excitatory barrages were observed. This was confirmed by recording from pairs of pyramidal cells separated by $>200-300 \mu \mathrm{m}$ (33 paired recordings) (Fig. 2A). Synaptic barrages were always synchronized in the paired recordings, the earliest excitatory barrages in the leading cell (Fig. $2 \mathrm{~A}$, red trace) being coincident with inhibitory barrages in the other, as indicated by the cross-correlogram minima being close to 0 time delay (Fig. $2 B$ ). The late excitatory barrages in both cells were also synchronous (Fig. 2C), and the event terminated simultaneously in both neurons. The velocity of the epileptiform wave, calculated from the delay in the time to the 
A. Occipital neocortex: coronal slice

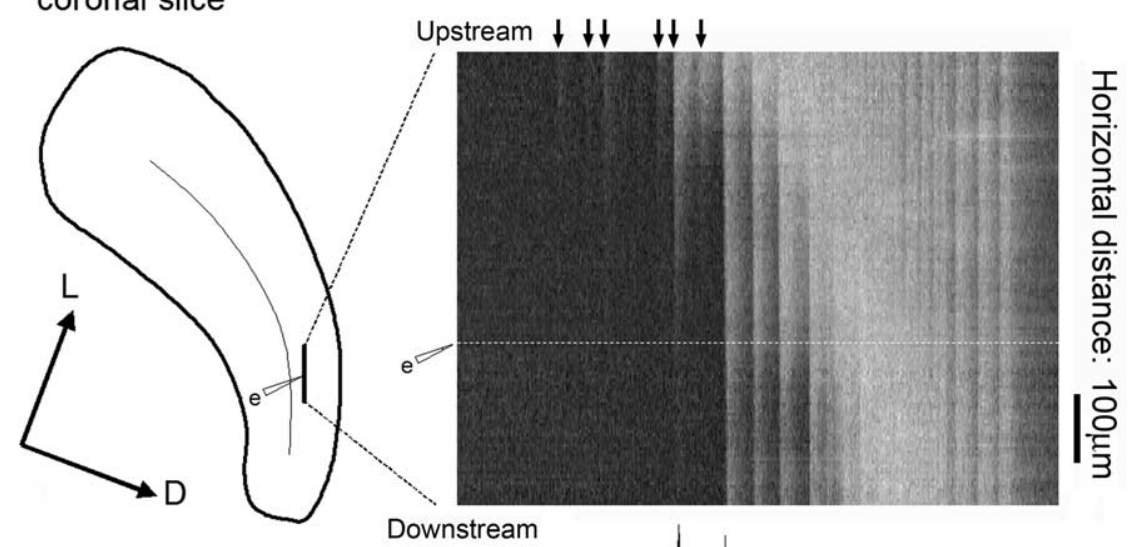

Downstream

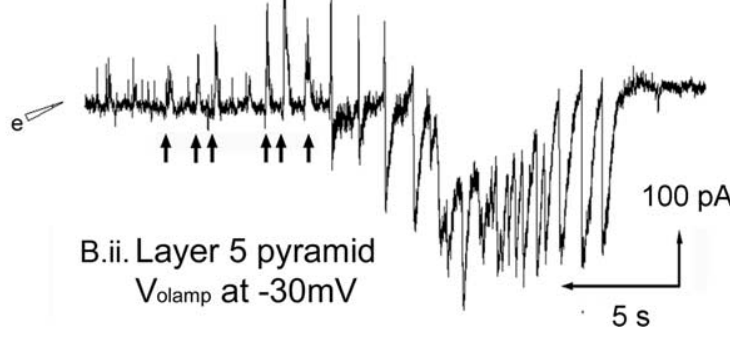

Figure 1. The slow stepwise propagation of $0 \mathrm{Mg}^{2+}$ epileptiform events reveals prominent feedforward inhibition. $\boldsymbol{A}$, Schematic of a coronal slice showing the recording electrode (labeled e) and the location of a line scan through layer 5 . Imaging was done at $30 \mathrm{~Hz}$ using a $10 \times$ objective. L, Lateral; $\mathrm{D}$, dorsal. Bi, Line scan showing the neuropil $\mathrm{Ca}^{2+}$ fluorescence as an epileptiform event progresses from top to bottom; distance is plotted on the ordinate, and time is on the abscissa. Each row of pixels represents the neuropil $\mathrm{Ca}^{2+}$ fluorescence from a bin $40 \times 4 \mu \mathrm{m}$ (165 bins is equivalent to $660 \mu \mathrm{m}$ total length). The location of the recording electrode (labeled e) is shown. Bii, The $V_{\text {clamp }}$ recording from the layer 5 pyramidal cell plotted below on the same timescale. The cell was held at $-30 \mathrm{mV}$, approximately halfway between the reversal potentials for GABA and glutamate, to distinguish inhibitory drives (upward deflections) and excitatory drives (downward deflections). Note the prominent inhibitory volleys (arrowed) corresponding with upstream activity in the line scan.

\section{Paired $V_{\text {clamp }}$ recordings at $-30 \mathrm{mV}$ : Layer 5 pyramids: $395 \mu \mathrm{m}$ separation}
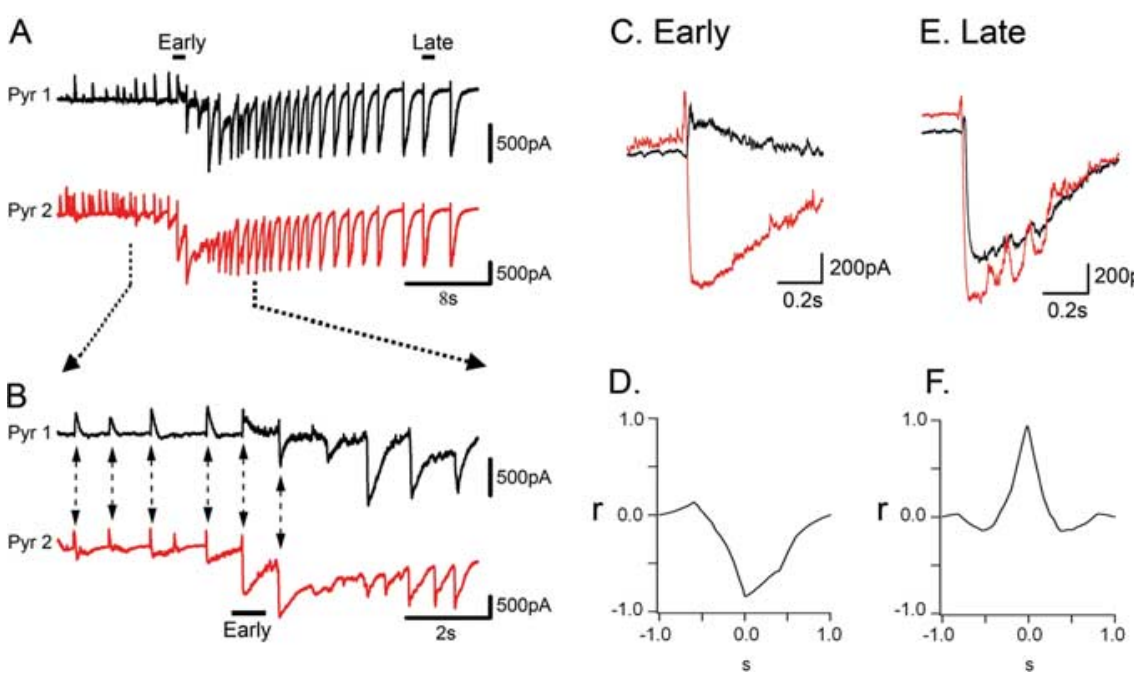

Figure 2. Correlated synaptic barrages in layer 5 pyramidal cells. $A, V_{\text {clamp }}$ recordings from a pair of layer 5 pyramids (Pyr 1, Pyr 2) located $395 \mu \mathrm{m}$ apart. $\boldsymbol{B}$, An expanded view of same recording. The arrows highlight the correspondence between excitatory drive in the earlier activated cell and inhibition of later activated cell. $\boldsymbol{C}, \boldsymbol{D}$, Enlarged view of one of the early synaptic volleys in the event, showing the tight temporal correlation between the excitation in the earlier activated cell with the inhibition in the later activated one. The correlation coefficient achieves its maximal negative value close to 0 time difference. $\boldsymbol{E}, \boldsymbol{F}$, The late barrages also show a high temporal correlation, and, because they are both predominantly excitatory, there is a peak in the correlation coefficient close to 0 . peak excitatory drive in these separate cell recordings, was $119.2 \pm 14.9 \mu \mathrm{m} / \mathrm{s}$ (mean \pm SEM; $n=10$ paired recordings; mean separation, $589 \pm 91 \mu \mathrm{m})$.

How do these inhibitory barrages influence the recruitment of cells to an ictal event? To answer this, we made use of a previous observation that adjacent pyramidal cells experience almost identical synaptic barrages during the ictal event ( $n=6$ pairs) (Fig. $3 A$ ). This presented an opportunity to assess the input-output function of these cells during the critical recruitment phase (Fig. 3B): we recorded from pairs of adjacent layer 5 pyramids holding one cell in $V_{\text {clamp }}$ at $-30 \mathrm{mV}$ to monitor the network barrages, while holding the other in $I_{\text {clamp }}$ mode. Remarkably, pyramids can resist firing for prolonged periods of time at the start of the event despite experiencing a dramatic shift in the apparent excitatory-inhibitory (E-I) balance, toward excitation. We confirmed that this period of paradoxical quiescence was not an artifact of the whole-cell recording mode by repeating these experiments monitoring the firing rate of the second cell in cell-attached mode (Fig. 3C).

To determine the mechanism underlying the delayed recruitment, we chose for additional analysis three paired recordings from adjacent layer 5 pyramids that showed particularly protracted periods of excitation before the first intense firing output (Fig. $2 C, D$, one $V_{\text {clamp }} / I_{\text {clamp }}$ pair and two $V_{\text {clamp }} /$ cell-attached pairs). We examined $200 \mathrm{~ms}$ bins centered on successive excitatory peaks (minima) in the $V_{\text {clamp }}$ recording because we assumed that the peak probability of firing would coincide with the peak inward current [this assumption was confirmed by our ictal simulations (see Fig. 5)]. During these $200 \mathrm{~ms}$ bins, we counted the number of actions potentials in the output neuron $\left(I_{\text {clamp }} /\right.$ cell-attached cell), the maximum E-I shift ( $V_{\text {clamp }}$ minima minus baseline), and finally an index of inhibition, the integral of the $d I / d t$ plot that was in excess of 5 SDs above the mean $d I / d t$ (supplemental Fig. 2, available at www.jneurosci.org as supplemental material; the analysis was repeated using 3 SDs as the cutoff and giving the same general result). This final index made use of the defining feature of the inhibitory barrages: their fast, large-amplitude upward deflection.

The action potential trains were notable for showing an abrupt switch from quiescence to intense firing (Fig. $3 B, C$, arrows in bottom). We divided the event into an early "low output" period and a 


\section{Paired recordings of adjacent pyramids}
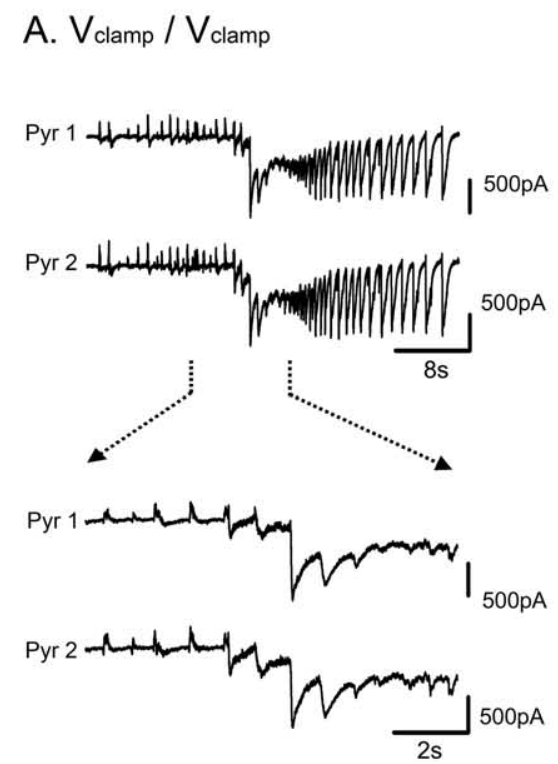

B. V $V_{\text {clamp }} / I_{\text {clamp }}$
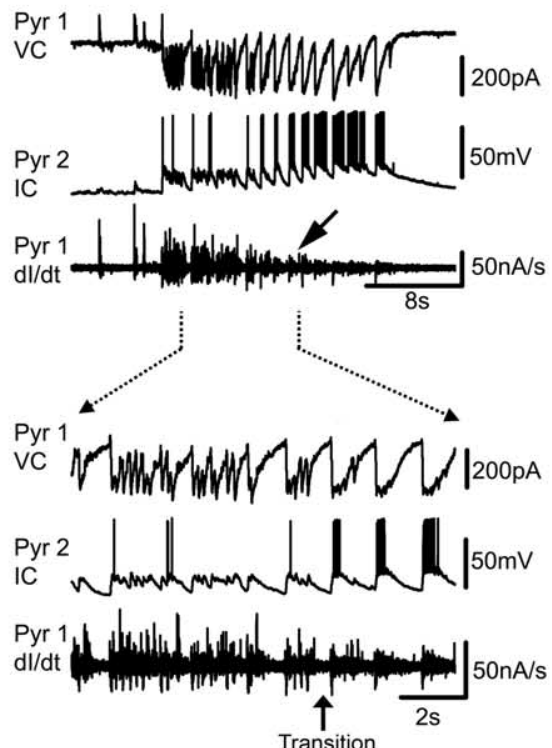

C. Vclamp / cell attached
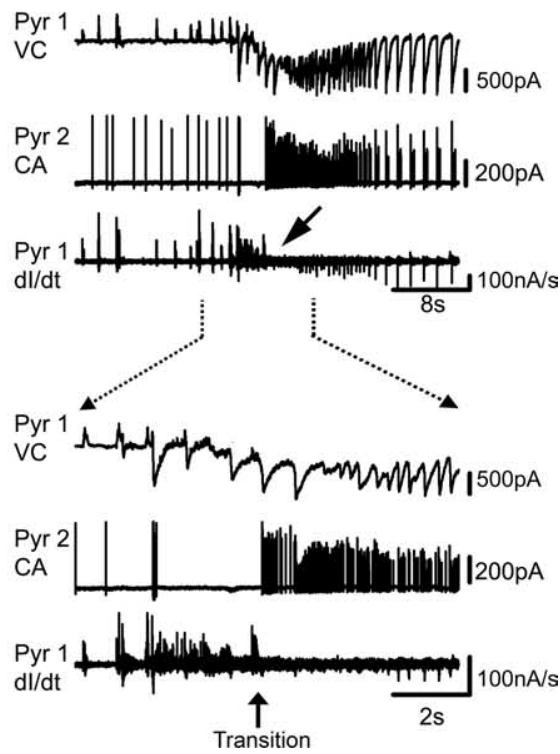

Figure 3. Input- output functions from layer 5 pyramid cells during epileptiform events indicate an inhibitory veto. $A$, Paired $V_{\text {clamp }}$ recordings from adjacent layer 5 pyramidal cells show that adjacent cells experience almost identical synaptic volleys during the epileptiform events. The bottom panels show an expanded view of the same recordings. $\boldsymbol{B}$, Paired $V_{\text {clamp }}$ and $I_{\text {clamp }}$ recordings from adjacent layer 5 pyramidal cells. Early in the event, pyramid 2 fires only occasionally despite the $V_{\text {clamp }}$ recording showing relatively intense excitatory barrages. The derivative of the $V_{\text {clamp }}$ recordings $(d / / d t)$ highlights the role of the large-amplitude inhibitory volleys, showing that the development of intense firing during the paroxysmal depolarizations occurs only as the inhibitory volleys wane (arrow). C, Paired recordings from adjacent layer 5 pyramids, with one cell being held in $V_{\text {clamp }}$ mode, whereas the second cell is recorded in cell-attached mode. This preserves the physiological integrity of the cell better than whole-cell current clamp yet still permits a high quality recording of the action potential trains. Like the $V_{\text {clamp }} / I_{\text {clamp }}$ pairs, these recordings show an abrupt transition to firing synchronous with the cessation of the intense inhibitory restraint, as indicated by the sudden change in the $d / / d t$ trace (arrow). Pyr 1, Pyramidal cell $1 ;$ Pyr 2, pyramidal cell 2.

late ictal period, defining the transition by the dramatic jump in the number of action potentials in the $200 \mathrm{~ms}$ bin [average \pm SEM number of action potentials in the $200 \mathrm{~ms}$ windows for the five bursts before the transition, $1.79 \pm 0.63(n=14)$; for the five bursts after the transition, $8.47 \pm 0.80(n=15)]$ (Fig. $4 A i, B i)$. First, we confirmed the paradoxical nature of the low output period by measuring the mean E-I shift for 20 action potentials during baseline (interictal) periods. For these three paired recordings, the mean E-I shift during the low output period was 16.4-fold greater than for the interictal action potentials (Fig. 4 Aii, dotted line), yet the mean number of action potentials in these 200 ms bins was just 0.7 ( 49 action potentials in 70 bins; this represented the total output of the cells because they did not fire out with these bins). Clearly, the output of the cell during this period did not simply reflect the E-I balance, as crudely assessed by the voltage-clamp recording.

The defining feature of the low output period was the initially intense degree of inhibition and its gradual dissolution. Analysis of the key excitatory barrages on either side of the transition period (20 barrages before and 10 after) showed that there was just a 1.22-fold excitatory shift in the E-I balance from before the transition to afterward but a 2.42 -fold drop in our index of inhibition (which could also contribute to the excitatory shift in the $\mathrm{E}-\mathrm{I}$ balance). Importantly, the change in E-I balance was a progressive increase over the 30 barrages spanning the transition (Fig. 4 Bii,Cii), whereas the changes in the number of action potentials and the degree of inhibition were both abrupt and simultaneous (Fig. 4C). We concluded from this that the transition came about not from an increase in excitatory drive but rather a collapse in the inhibitory restraint.
We wanted to get a better understanding of the synaptic drive during these early barrages and also the previous inhibitory barrages. To do this, we attempted to negate the inhibitory drive by holding the pyramidal cells at the reversal potential for $\mathrm{GABA}_{\mathrm{A}}$ $\left(E_{\mathrm{GABA}}\right)$. We determined $E_{\mathrm{GABA}}$ by recording multiunit IPSCs triggered extracellularly while blocking AMPA, NMDA, and $\mathrm{GABA}_{\mathrm{B}}$ synaptic transmission pharmacologically (Fig. $5 A$ ). We then made voltage-clamp recordings from adjacent pairs of layer 5 pyramidal cells, holding one at $-30 \mathrm{mV}$ as in previous recordings and the other at $E_{\mathrm{GABA}}(-59.3 \mathrm{mV})$ (Fig. $\left.5 B\right)$. Although there are inherent difficulties in achieving a good voltage clamp of cells experiencing the level of synaptic bombardment seen during these barrages, we were helped in our aim of trying to negate the inhibitory drive by the fact that the key inhibitory synapses are likely to be those located on the soma and proximal dendrites (Trevelyan and Watkinson, 2005) and therefore that these synapses should be well clamped. As such, we believe that the voltage-clamp recordings at $-59 \mathrm{mV}$ gave a reasonable approximation of the amplitude of the excitatory drive during these barrages.

These paired recordings clearly show that, in the $-30 \mathrm{mV}$ voltage-clamp recordings, the early inhibitory barrages occlude an enormous excitatory drive. The amplitude of the excitatory drive was readily apparent in the $-59 \mathrm{mV}$ recordings, in which the average peak inward current was in excess of $1 \mathrm{nA}$ during these preictal events. There was a crescendo in the amplitude of these inward currents from the initial barrages up to the time when our imaging experiments suggest that the ictal event invades the cortical column (Fig. 1). Importantly, however, the amplitude of the excitatory drive during preictal events was as 
A.

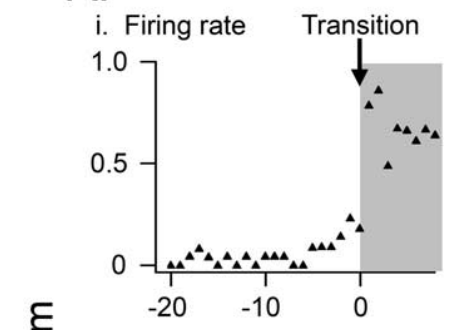

ii. Excitatory-inhibitory balance

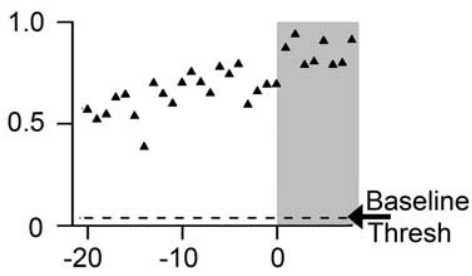

iii. Inhibition

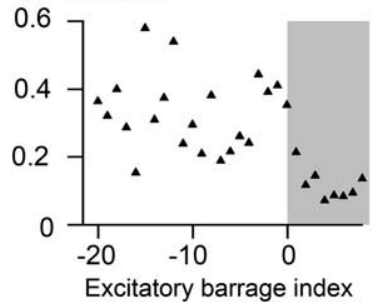

B.

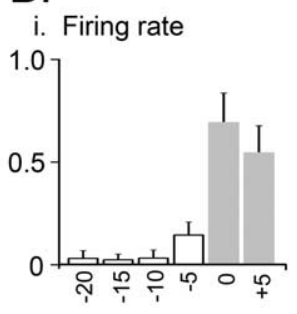

ii. E-I balance

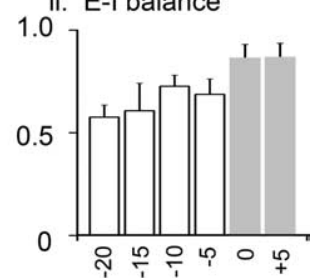

iii. Inhibition

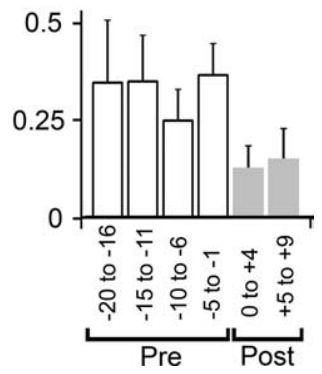

Figure 4. The transition coincides with a sharp drop in the index of inhibition. Analysis of the excitatory barrages either side of the transition from paradoxical quiescence to intense bursting for three recordings from adjacent pyramids, in which there was a long delay between the first excitatory barrages and the first action potential burst. We analyzed $200 \mathrm{~ms}$ bins around the peaks of the inward currents in the $V_{\text {clamp }}$ recordings, to derive the number of action potentials in the $I_{\text {clamp }} /$ cell-attached recorded cell in that bin $(i)$, the peak excitatory shift in the holding current in the $V_{\text {clamp }}$ cell (ii), and the degree of inhibition as assessed by the $d / / d t$ plots (iii) (for more details, see Results). $\boldsymbol{B i}$, The mean \pm SEM firing rate (normalized) for the 20 excitatory bursts immediately preceding the transition and the $10 \mathrm{immediately}$ after. Bii, Equivalent plot for the peak excitatory shift in the holding current for the $V_{\text {clamp }}$ cell. Biii, Same plot showing the drop in inhibition at the time of the transition.

large as many of the post-ictal afterdischarges, yet the output of pyramidal cells at these two time points is radically different.

We averaged the excitatory drive from five consecutive preictal barrages (events that were coincident with predominant inhibitory barrages in the other cell held at $-30 \mathrm{mV}$ ) and then injected this same current profile back into pyramidal cells to get an appreciation of how powerful the excitatory drive is in the absence of inhibition. The cells were given a small constant depolarizing current to get the resting membrane potential at -59 $\mathrm{mV}$, so that the current injection was at the same baseline membrane potential at which it was recorded. We then injected reduced versions of the current profile to determine what level of current produced action potentials in half of the trials (threshold). This threshold was achieved when injecting just $9.9 \pm 1.4 \%$ (mean \pm SEM; range of $5-14 \% ; n=7$ cells) of the excitatory current. Thus, the excitatory drive is typically $\sim 10$ times greater than that normally required to achieve threshold for firing in these cells, yet, during these preictal barrages, the cells frequently do not fire or fire only a single action potential. When we injected the full current, the cells fired a barrage of action potentials, very like their behavior during a full ictal event. We concluded from

these experiments that, during preictal barrages, the inhibition successfully vetoes an extremely powerful excitatory drive.

\section{Disinhibition models of epileptiform propagation}

It was important to reconcile this model of epilepsy with previous studies of epileptiform propagation, which used low doses of $\mathrm{GABA}_{\mathrm{A}}$ antagonists to partially disinhibit brain slices (ChagnacAmitai and Connors, 1989; Pinto et al., 2005). We therefore applied low concentrations of the $\mathrm{GABA}_{\mathrm{A}}$ antagonist PTX at the same time that we washed out the $\mathrm{Mg}^{2+}$ ions, while recording layer 5 pyramidal cells in voltage clamp at $-30 \mathrm{mV}$ to monitor the inhibitory barrages (Fig. 6). We found that the same concentrations of PTX as used in the previous disinhibition studies (2.5-10 $\mu \mathrm{M})$ (Pinto et al., 2005) effectively nullified the inhibitory barrages. In all experiments $(n=13)$, except one using $2.5 \mu \mathrm{M}$ PTX (Fig. 6A shows this single example, in which the inhibitory barrages were preserved), the preictal synaptic bursts were overwhelmingly excitatory (Fig. $6 B, C$ ). Paired recordings from separate pyramidal cells showed that, like the simple disinhibition models (Chagnac-Amitai and Connors, 1989; Pinto et al., 2005), ictal events in $0 \mathrm{Mg}^{2+}$ and $5 \mu \mathrm{M}$ picrotoxin propagate over two orders of magnitude faster than those in $0 \mathrm{Mg}^{2+}$ alone (supplemental Fig. 3, available at www.jneurosci.org as supplemental material)

These experiments suggest a threshold at $\sim 2.5 \mu \mathrm{M}$ PTX for blockade of this critical inhibitory component (Fig. 6D). This is remarkably close to the minimum PTX concentration $(2-4 \mu \mathrm{M})$ required to trigger propagating activity in the partial disinhibition model (Pinto et al., 2005). This presumably explains why the pattern of propagation seen in our studies differs from the smoother and far more rapid ( $\sim 200-300$ times faster) propagation in all disinhibition models. In contrast, our results are completely consistent with measures of propagation velocity in a previous study of the $0 \mathrm{Mg}^{2+}$ model in adult guinea pigs (Wong and Prince, 1990).

\section{Imaging of ictal recruitment}

Having examined how individual neurons are recruited to an ictal event, we wanted to understand how populations of neurons are recruited, at the network level. To achieve this, we performed fast imaging of OGB1 bulk-loaded slices at higher magnification ( $20 \times$ objective) (supplemental movie, available at www.jneurosci.org as supplemental material). We calculated the derivative of their neuronal somatic $\mathrm{Ca}^{2+}$ fluorescent signal $(d \mathrm{CS} / d t)$ because this was found to represent accurately the action potential trains during the recruitment period (Fig. 7A).

The $d \mathrm{CS} / d t$ traces for each neuron had multiple peaks (Fig. $7 B i$ ), indicating that recruitment was staggered over several synaptic barrages. This was consistent with our electrophysiological recordings. The peaks had a periodicity of $\sim 0.5-2 \mathrm{~Hz}$ for all cells in all movies analyzed, the same periodicity as the preictal bursts recorded in $V_{\text {clamp. }}$.

The most striking feature of the $d \mathrm{CS} / d t$ grayscale plots, however, was that the individual peaks for all neurons were synchronized to within $100 \mathrm{~ms}$ (Fig. 7Bi). This synchronicity indicates that neuronal recruitment is intermittent, occurring only at specific time points (additional examples shown in supplemental Fig. 4, available at www.jneurosci.org as supplemental material). Furthermore, the activity pattern of every neuron is coordinated with these bursts from the earliest time at which a change in activity is detectable with $\mathrm{Ca}^{2+}$ imaging. We concluded from this that the progression of epileptiform activity is best viewed as a 
series of crises, at which times cells are either recruited to the event or resist by virtue of the inhibitory restraint.

The episodic nature of neuronal recruitment to an event permits a simple clustering of the neurons based on the timing of the maximum $d \mathrm{CS} / d t$ (Fig. $7 \mathrm{Bii}$ ). Our analysis was limited to an examination of layers 4 and 5 because of the restricted field of view. Even so, the clustering showed a notable spatial pattern. Layer 5 neurons were recruited before those in layer 4 (Fig. 7Biii) and occasionally tens of seconds in advance. In these latter slices with especially delayed recruitment, many of the layer 5 neurons showed a slow ratcheting increase in $\mathrm{Ca}^{2+}$ signal over the time course of many synaptic barrages (supplemental Fig. 5, available at www.jneurosci. org as supplemental material). In contrast, layer 4 cells usually were recruited over the course of a smaller number of bursts, usually with one jump in the $\mathrm{Ca}^{2+}$ signal that was much larger than the other steps. Perhaps for this reason, the spatial clustering based on a single maximum usually appeared more precise for layer 4 than for layer 5 . The spatial periodicity of the layer 4 clustering was $\sim 150-250 \mu \mathrm{m}$.

\section{Discussion}

We have shown here that epileptiform propagation in a widely used in vitro model of epilepsy occurs as a slow, stepwise recruitment of modules of cortex. Electrophysiological recordings suggest strongly that the timing of pyramidal recruitment to an ictal event is determined primarily by the efficacy of a powerful inhibitory restraint.

There are two critical observations. First, in slices with particularly protracted recruitment of pyramidal cells, paired recordings indicate that the recruitment and the failure of inhibition are effectively coincident. Second, and perhaps more importantly, the inhibitory barrages occlude an excitatory drive that is extremely powerful from the very earliest stages of the event. Thus, even at the time when the barrages are seen as intense upward deflections in the $-30 \mathrm{mV}$ traces, the cells are experiencing very powerful excitation. It is not that the inhibition extends farther forward than the excitation, rather that the inhibition vetoes the excitation. Together with the imaging data, these results suggest that the modules are defined by this inhibitory element.

\section{Activity propagation in other neocortical preparations}

Such are the demands on this inhibitory drive that disinhibiting the slice with very low doses of PTX $(2.5 \mu \mathrm{M})$ effectively nullifies the restraint and presumably explains why epileptiform events in $>2.5 \mu \mathrm{M}$ PTX propagate $\sim 200-300$ times faster (Chervin et al., 1988; Wadman and Gutnick, 1993; Telfeian and Connors, 1998, 1999; Pinto et al., 2005) than when inhibition is intact $\left(0 \mathrm{Mg}^{2+}\right.$ model). Notably, however, the speed of propagation of the early events when the slice is bathed in $\mathrm{Mg}^{2+}$-free solution (Wong and Prince, 1990) is comparable with that in the best known example of neocortical seizure propagation, the Jacksonian march (Jasper, 1969; Ohara et al., 2004). A similar transition, from slow propagation in $0 \mathrm{Mg}^{2+}$ to fast synchronization when the slice is also absence of any inhibition.
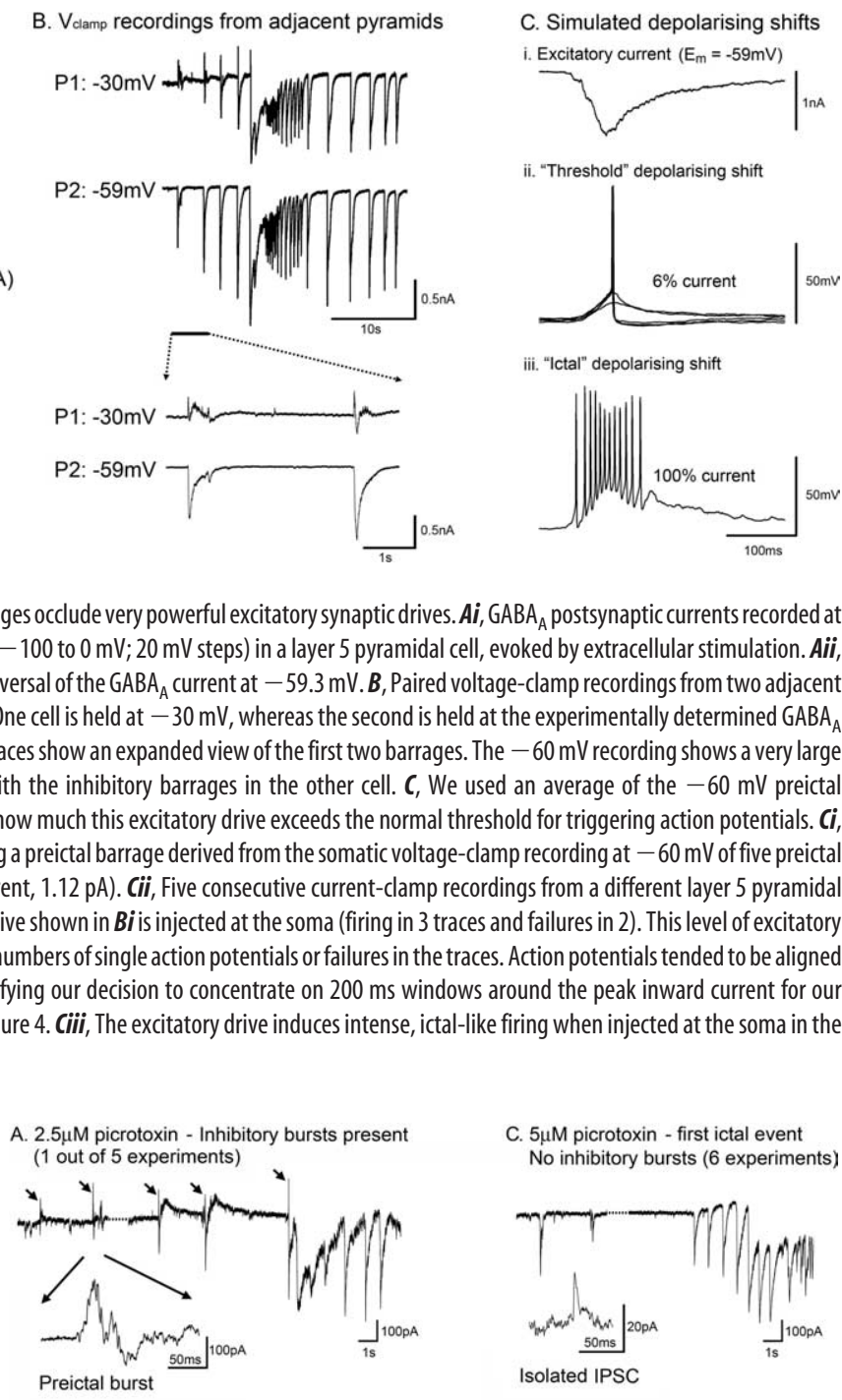

$A i$ GABA ${ }_{A}$ postsynaptic currents recorded at six different holding potentials ( -100 to $0 \mathrm{mV} ; 20 \mathrm{mV}$ steps) in a layer 5 pyramidal cell, evoked by extracellular stimulation. Aii, Current-voltage plot showing reversal of the $\mathrm{GABA}_{A}$ current at $-59.3 \mathrm{mV}$. $\boldsymbol{B}$, Paired voltage-clamp recordings from two adjacent

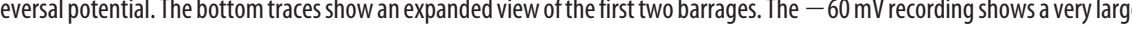
Average excitatory current during a preictal barrage derived from the somatic voltage-clamp recording at $-60 \mathrm{mV}$ of five preictal hen $6 \%$ of the excitatory drive shown in Bi is injected at the soma (firing in 3 traces and failures in 2). This level of excitatory cosely to the peak current, justifying our decision to concentrate on $200 \mathrm{~ms}$ windows around the peak inward current for previous analyses depicted in Figure 4. Ciii, The excitatory drive induces intense, ictal-like firing when injected at the soma in the
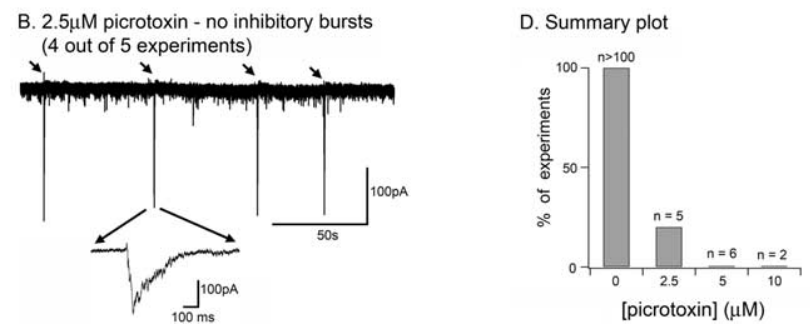

Figure 6. Inhibitory barrages are effectively nullified by low doses of picrotoxin. $A, B$, Voltage-clamp recordings from layer 5 pyramidal cells held at $-30 \mathrm{mV}$ when $2.5 \mu \mathrm{m}$ PTX is bath applied simultaneously with the washout of $\mathrm{Mg}^{2+}$ ions. $A$ shows the single example in which the inhibitory barrages were reasonably preserved at this PTX concentration. The inset shows an expanded view of a preictal burst. $\boldsymbol{B}$ shows four preictal bursts from a more typical example, with the first burst shown at an expanded timescale in the inset. The characteristic upward deflections (see arrows in $\boldsymbol{A}$ ) in the voltage-clamp trace are absent, being replaced by episodic, massive excitatory barrages (arrows). C, Synaptic barrages in $5 \mu \mathrm{m}$ PTX; several preictal events are shown as well as the first full ictal event recorded. Again, the upward deflections are absent. Inhibitory barrages are never seen with 5 or $10 \mu \mathrm{M}$ (data not shown) picrotoxin, despite the occurrence of isolated IPSC being readily apparent during quiescent periods in all recordings (inset). D, Summary plot showing the proportion of experiments at the different levels of picrotoxin that displayed inhibitory barrages. These preictal inhibitory barrages were always seen in $0 \mathrm{Mg}^{2+}$ in the absence of $\mathrm{GABA}_{\mathrm{A}}$ blockers. 


\section{A. Derivation of dCS/dt plots}

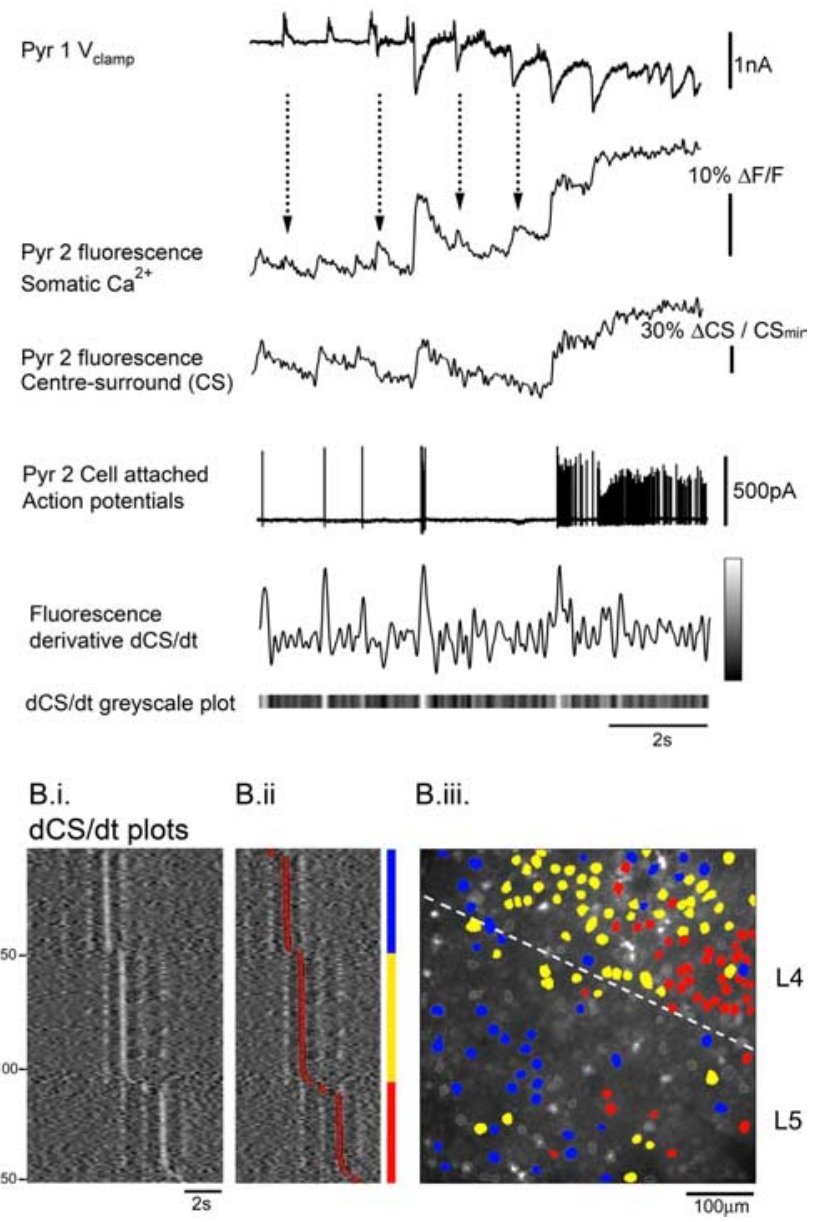

Figure 7. Somatic $\mathrm{Ca}^{2+}$ fluxes reveal episodic localized recruitment. $\boldsymbol{A}$, The derivation of the cellular $\mathrm{Ca}^{2+}$ signal. We recorded from adjacent pyramids (Pyr 1, Pyr 2). Pyr 1 was recorded in $V_{\text {clamp }}$ mode to monitor the local synaptic activity. Pyr 2 was a labeled cell that we recorded in cell-attached mode to allow the $\mathrm{Ca}^{2+}$ signal to be related to the action potential train. The simple somatic $\mathrm{Ca}^{2+}$ signal shows several prominent deflections that reflect the synaptic barrage (dashed line arrows) but importantly do not reflect the action potential train. Subtracting the neuropil signal removes these artifactual deflections (third trace). The derivative of the signal highlights the sudden increases in fluorescent signal, which reflect the sudden recruitment of neurons. The bottom panel shows how this derivative trace is transformed into the grayscale plots shown in $\boldsymbol{B}$, which shows grayscale representations from 152 identified neurons. $\mathbf{B i}$, Grayscale plot of the temporal derivatives $(d C S / d t)$ of the center-surround signals from 152 neurons ordered by the time of the maximum for each trace. Each row of pixels represents a $d C S / d t$ trace: high rate of change of fluorescence is depicted as lighter gray. Each trace has several peaks. Bii, The same plot, with the single maximum rate of change for each cell marked (red). This ordering clearly shows three main clusters of cells. They are color-coded as shown, and their positions are indicated in the photomicrograph in Biii. Note the tight and generally non-overlapping spatial distributions of the yellow and red groups in layer 4. The lamina borders were determined from the location of the filled cells after post hoc staining for biocytin and matching this up to the electrode location in the field of view (the faint triangular shadow of one of the electrodes can just be seen at the bottom right corner of the field of view). Movie was taken using a $20 \times$ objective.

disinhibited, has also been noted in the entorhinal cortex and dentate gyrus (Heinemann et al., 1992).

Our imaging experiments used juvenile animals (postnatal day 13-17) primarily because loading of cortical slices with $\mathrm{Ca}^{2+}$ dyes is best at these ages. Although undoubtedly the cortex is not fully mature at this stage, we believe for a number of reasons that these epileptiform activity patterns are adult like and not related to neonatal giant depolarizing potentials (also known as early network oscillations) (Ben-Ari et al., 1989; Garaschuk et al., 2000). First, these latter, physiological patterns of activity become vanishingly rare after approximately postnatal day 7 (Garaschuk et al., 2000), as the $E_{\mathrm{GABA}}$ becomes progressively more hyperpolarized. Second, we confirmed that the same electrophysiological patterns of activity are also found in slices from adult animals (postnatal days 30, 31, and 49) (supplemental Fig. 6, available at www.jneurosci.org as supplemental material). Third, a previous study of the same epilepsy model in adult guinea pig brain slices using paired extracellular electrodes to record propagating epileptiform activity reported the same speed of propagation as we found. The fact that the modular nature of the propagation was not apparent in these paired recordings highlights the power of using $\mathrm{Ca}^{2+}$ imaging to visualize network activity.

\section{In vivo patterns of epileptic activity}

In vitro slice preparations of neocortex clearly are impoverished models of the natural in vivo state, but the importance of these preparations is that they allow experiments that are difficult or impossible in vivo. One such experiment is paired recordings of adjacent neurons, which allowed us to correlate the dissolution of the inhibitory restraint with the transition from quiescence to intense firing. The $V_{\text {clamp }}$ recordings at $-30 \mathrm{mV}$ were critical for showing this, but as far as we are aware, no one has recorded $V_{\text {clamp }}$ traces at $-30 \mathrm{mV}$ during epileptic activity in vivo. Currentclamp recordings, however, do provide a common point of reference between our studies and the in vivo situation.

The veto inhibition caused a very characteristic $I_{\text {clamp }}$ trace, with regular large depolarizations accompanied by infrequent action potentials, and this is strikingly similar to slow waves recorded in neocortical cells in vivo before their recruitment (Timofeev and Steriade, 2004). Furthermore, in vivo whole-cell recordings with different levels of intracellular $\mathrm{Cl}^{-}$indicate a large inhibitory contribution to these slow waves preceding the fast runs (Timofeev et al., 2002) and critically that the transition into the fast runs coincides with a change in the pattern of inhibition, just as our in vitro experiments. Finally, both in vivo electrophysiological (Prince and Wilder, 1967; Goldensohn and Salazar, 1986; Timofeev et al., 2002) and imaging (Schwartz and Bonhoeffer, 2001; Schwartz, 2003) recordings have provided evidence for a restraining inhibition opposing epileptic spread: focal interictal activity is synchronous with suppressed activity in the surrounding territory. Activity escaped from the focus only after the gradual dissolution of the inhibitory annulus, and, even then, there was evidence for reduced activity in front of the wave (Schwartz and Bonhoeffer, 2001).

There appears then to be sufficient commonality between the in vitro and in vivo states to indicate that our view of modular propagation regulated by inhibition may be the basis for the demonstrably slow Jacksonian march and may be related to the well described phenomenon of surround inhibition (Prince and Wilder, 1967; Schwartz and Bonhoeffer, 2001).

Chandelier cells have been proposed previously to have a vetoing role, although recent work has cast this into doubt (Szabadics et al., 2006). Another candidate for this role is the basket cell population, particularly given the remarkable arrangement of synaptic inputs to the proximal structures in pyramidal cells: pyramidal somata and proximal dendrites receive a dense inhibitory innervation and yet are completely devoid of any excitatory drive. Theoretical considerations indicate that this arrangement is well suited to provide vetoing inhibition (Trevelyan and Watkinson, 2005), consistent with a previous experimental demonstration of veto inhibition for an analogous anatomical ar- 
rangement regulating the escape reflex in crayfish $(\mathrm{Vu}$ and Krasne, 1992). If this differential pattern of inhibition does underlie the feedforward inhibition we described here, then this theoretical model would predict that there would be a preferential early recruitment of interneurons during an ictal event. Notably, several groups have shown exactly this pattern of early activation of fast-spiking interneurons in both frontal cortical (Kawaguchi, 2001) and hippocampal (Ziburkus et al., 2006) in vitro preparations, as well as in vivo (Timofeev et al., 2002). Such a view suggests that the earliest recruited cells in our preparation are also interneurons, although our inability to distinguish neuronal subpopulations in network $\mathrm{Ca}^{2+}$ imaging experiments prevents additional examination at this time. If there is an uneven laminar distribution of these early recruited interneurons, this might also explain the apparent difference in the degree of spatial segregation seen in layers 4 and 5 .

We have not addressed in this study why the inhibitory drive fails. The nature of the failure, however, is clearly an important issue. There are a number of possible mechanisms for which there already exists some experimental evidence: synaptic depression (Kraushaar and Jonas, 2000) by several possible presynaptic or postsynaptic mechanisms (Larkman and Jack, 1995) such as reduction in the probability of vesicular release induced by either presynaptic $\mathrm{GABA}_{\mathrm{B}}$ activation (Giustizieri et al., 2005) or metabotropic glutamate receptor activation (Giustizieri et al., 2005; Qian and Noebels, 2006), depletion of transmitter stores (Bekkers and Stevens, 1990), or receptor desensitization (Thompson and Gahwiler, 1989b); failure of interneuronal firing, possibly by going into a depolarizing block (Ziburkus et al., 2006); and shift in the $\mathrm{GABA}_{\mathrm{A}}$ reversal potential (Thompson and Gahwiler, 1989a; Staley et al., 1995; Jarolimek et al., 1999). These different mechanisms are not mutually exclusive, and it seems quite probable that the reason why the inhibitory restraint fails involves a combination of several of these. The fact that we demonstrated a means of addressing this issue in an in vitro preparation, in which one has more control over the experimental design than often is the case in vivo, means that this important and difficult question may now prove more tractable.

\section{Conclusion}

The suppression of epileptiform activity is clearly an extreme test of the power of lateral inhibition and implies that this inhibition could similarly veto less intense, physiological activity. Our results show that the collapse of the inhibition determines the timing of recruitment of cells to an epileptiform event and, second, that recruitment occurs in spatial clusters. They further suggest that the interictal to ictal transition is crucially controlled by this inhibitory veto, which could be targeted therapeutically. Together, these results suggest that inhibition defines cortical modules. These territories are most clearly delineated in layer 4, in which they are regularly spaced and of the right dimensions to suggest that the modules may be equivalent to cortical columns.

\section{References}

Bekkers JM, Stevens CF (1990) Presynaptic mechanism for long-term potentiation in the hippocampus. Nature 346:724-729.

Ben-Ari Y, Cherubini E, Corradetti R, Gaiarsa JL (1989) Giant synaptic potentials in immature rat CA3 hippocampal neurones. J Physiol (Lond) 416:303-325.

Chagnac-Amitai Y, Connors BW (1989) Horizontal spread of synchronized activity in neocortex and its control by GABA-mediated inhibition. J Neurophysiol 61:747-758.

Chervin RD, Pierce PA, Connors BW (1988) Periodicity and directionality in the propagation of epileptiform discharges across neocortex. J Neurophysiol 60:1695-1713.
Connors BW (1984) Initiation of synchronized neuronal bursting in neocortex. Nature 310:685-687.

Cossart R, Aronov D, Yuste R (2003) Attractor dynamics of network UP states in the neocortex. Nature 423:283-288.

Douglas RJ, Martin KA (2004) Neuronal circuits of the neocortex. Annu Rev Neurosci 27:419-451.

Garaschuk O, Linn J, Eilers J, Konnerth A (2000) Large-scale oscillatory calcium waves in the immature cortex. Nat Neurosci 3:452-459.

Gastaut H, Broughton R (1972) Epileptic seizures: clinical and electrographic features, diagnosis and treatment. Springfield, IL: Thomas.

Gilbert CD, Wiesel TN (1989) Columnar specificity of intrinsic horizontal and corticocortical connections in cat visual cortex. J Neurosci 9:2432-2442.

Giustizieri M, Bernardi G, Mercuri NB, Berretta N (2005) Distinct mechanisms of presynaptic inhibition at GABAergic synapses of the rat substantia nigra pars compacta. J Neurophysiol 94:1992-2003.

Goldensohn ES, Salazar AM (1986) Temporal and spatial distribution of intracellular potentials during generation and spread of epileptogenic discharges. Adv Neurol 44:559-582.

Heinemann U, Beck H, Dreier JP, Ficker E, Stabel J, Zhang CL (1992) The dentate gyrus as a regulated gate for the propagation of epileptiform activity. Epilepsy Res Suppl 7:273-280.

Hubel DH, Wiesel TN (1977) Ferrier lecture. Functional architecture of macaque monkey visual cortex. Proc R Soc Lond B Biol Sci 198:1-59.

Ikegaya Y, Aaron G, Cossart R, Aronov D, Lampl I, Ferster D, Yuste R (2004) Synfire chains and cortical songs: temporal modules of cortical activity. Science 304:559-564.

Jarolimek W, Lewen A, Misgeld U (1999) A furosemide-sensitive $\mathrm{K}^{+}-\mathrm{Cl}^{-}$ cotransporter counteracts intracellular $\mathrm{Cl}^{-}$accumulation and depletion in cultured rat midbrain neurons. J Neurosci 19:4695-4704.

Jasper HH (1969) Mechanisms of propagation: extracellular studies. In: Basic mechanisms of the epilepsies, including hippocampus (Jasper $\mathrm{HH}$, Ward AA, Pope A, eds). New York: Plenum.

Kawaguchi Y (2001) Distinct firing patterns of neuronal subtypes in cortical synchronized activities. J Neurosci 21:7261-7272.

Kraushaar U, Jonas P (2000) Efficacy and stability of quantal GABA release at a hippocampal interneuron-principal neuron synapse. J Neurosci 20:5594-5607.

Larkman AU, Jack JJ (1995) Synaptic plasticity: hippocampal LTP. Curr Opin Neurobiol 5:324-334.

MacLean JN, Watson BO, Aaron GB, Yuste R (2005) Internal dynamics determine the cortical response to thalamic stimulation. Neuron 48:811-823.

Malach R (1992) Dendritic sampling across processing streams in monkey striate cortex. J Comp Neurol 315:303-312.

Malach R, Amir Y, Harel M, Grinvald A (1993) Relationship between intrinsic connections and functional architecture revealed by optical imaging and in vivo targeted biocytin injections in primate striate cortex. Proc Natl Acad Sci USA 90:10469-10473.

Mody I, Lambert JD, Heinemann U (1987) Low extracellular magnesium induces epileptiform activity and spreading depression in rat hippocampal slices. J Neurophysiol 57:869-888.

Nimmerjahn A, Kirchhoff F, Kerr JN, Helmchen F (2004) Sulforhodamine 101 as a specific marker of astroglia in the neocortex in vivo. Nat Methods 1:31-37.

Ohara S, Ikeda A, Kunieda T, Yazawa S, Taki J, Nagamine T, Taki W, Miyamoto S, Hashimoto N, Shibasaki H (2004) Propagation of tonic posturing in supplementary motor area (SMA) seizures. Epilepsy Res 62:179-187.

Pinto DJ, Patrick SL, Huang WC, Connors BW (2005) Initiation, propagation, and termination of epileptiform activity in rodent neocortex in vitro involve distinct mechanisms. J Neurosci 25:8131-8140.

Prince DA, Jacobs K (1998) Inhibitory function in two models of chronic epileptogenesis. Epilepsy Res 32:83-92.

Prince DA, Wilder BJ (1967) Control mechanisms in cortical epileptogenic foci. "Surround" inhibition. Arch Neurol 16:194-202.

Qian J, Noebels JL (2006) Exocytosis of vesicular zinc reveals persistent depression of neurotransmitter release during metabotropic glutamate receptor long-term depression at the hippocampal CA3-CA1 synapse. J Neurosci 26:6089-6095.

Schwartz TH (2003) Optical imaging of epileptiform events in visual cortex in response to patterned photic stimulation. Cereb Cortex 13:1287-1298. 
Schwartz TH, Bonhoeffer T (2001) In vivo optical mapping of epileptic foci and surround inhibition in ferret cerebral cortex. Nat Med 7:1063-1067.

Silva LR, Amitai Y, Connors BW (1991) Intrinsic oscillations of neocortex generated by layer 5 pyramidal neurons. Science 251:432-435.

Staley KJ, Soldo BL, Proctor WR (1995) Ionic mechanisms of neuronal excitation by inhibitory GABAA receptors. Science 269:977-981.

Szabadics J, Varga C, Molnar G, Olah S, Barzo P, Tamas G (2006) Excitatory effect of GABAergic axo-axonic cells in cortical microcircuits. Science 311:233-235.

Telfeian AE, Connors BW (1998) Layer-specific pathways for the horizontal propagation of epileptiform discharges in neocortex. Epilepsia 39:700-708.

Telfeian AE, Connors BW (1999) Epileptiform propagation patterns mediated by NMDA and non-NMDA receptors in rat neocortex. Epilepsia 40:1499-1506.

Thompson SM, Gahwiler BH (1989a) Activity-dependent disinhibition. I. Repetitive stimulation reduces IPSP driving force and conductance in the hippocampus in vitro. J Neurophysiol 61:501-511.

Thompson SM, Gahwiler BH (1989b) Activity-dependent disinhibition. III. Desensitization and GABAB receptor-mediated presynaptic inhibition in the hippocampus in vitro. J Neurophysiol 61:524-533.
Timofeev I, Steriade M (2004) Neocortical seizures: initiation, development and cessation. Neuroscience 123:299-336.

Timofeev I, Grenier F, Steriade M (2002) The role of chloride-dependent inhibition and the activity of fast-spiking neurons during cortical spikewave electrographic seizures. Neuroscience 114:1115-1132.

Trevelyan AJ, Watkinson O (2005) Does inhibition balance excitation in neocortex? Prog Biophys Mol Biol 87:109-143.

Vu ET, Krasne FB (1992) Evidence for a computational distinction between proximal and distal neuronal inhibition. Science 255:1710-1712.

Wadman WJ, Gutnick MJ (1993) Non-uniform propagation of epileptiform discharge in brain slices of rat neocortex. Neuroscience 52:255-262.

Walther H, Lambert JD, Jones RS, Heinemann U, Hamon B (1986) Epileptiform activity in combined slices of the hippocampus, subiculum and entorhinal cortex during perfusion with low magnesium medium. Neurosci Lett 69:156-161.

Wong BY, Prince DA (1990) The lateral spread of ictal discharges in neocortical brain slices. Epilepsy Res 7:29-39.

Ziburkus J, Cressman JR, Barreto E, Schiff SJ (2006) Interneuron and pyramidal cell interplay during in vitro seizure-like events. J Neurophysiol 95:3948-3954. 